\title{
Assessment of Patellar Tendon Reflex Responses Using Second-Order System Characteristics
}

\author{
Brett D. Steineman, Pavan Karra, and Kiwon Park \\ Department of Mechanical and Aerospace Engineering, Trine University, Angola, IN 46703, USA \\ Correspondence should be addressed to Kiwon Park; parkk@trine.edu
}

Received 1 October 2015; Revised 13 January 2016; Accepted 14 January 2016

Academic Editor: Stefano Zaffagnini

Copyright ( 2016 Brett D. Steineman et al. This is an open access article distributed under the Creative Commons Attribution License, which permits unrestricted use, distribution, and reproduction in any medium, provided the original work is properly cited.

\begin{abstract}
Deep tendon reflex tests, such as the patellar tendon reflex (PTR), are widely accepted as simple examinations for detecting neurological disorders. Despite common acceptance, the grading scales remain subjective, creating an opportunity for quantitative measures to improve the reliability and efficacy of these tests. Previous studies have demonstrated the usefulness of quantified measurement variables; however, little work has been done to correlate experimental data with theoretical models using entire PTR responses. In the present study, it is hypothesized that PTR responses may be described by the exponential decay rate and damped natural frequency of a theoretical second-order system. Kinematic data was recorded from both knees of 45 subjects using a motion capture system and correlation analysis found that the mean $R^{2}$ value was 0.99 . Exponential decay rate and damped natural frequency ranges determined from the sample population were -5.61 to -1.42 and $11.73 \mathrm{rad} / \mathrm{s}$ to $14.96 \mathrm{rad} / \mathrm{s}$, respectively. This study confirmed that PTR responses strongly correlate to a second-order system and that exponential decay rate and undamped natural frequency are novel measurement variables to accurately measure PTR responses. Therefore, further investigation of these measurement variables and their usefulness in grading PTR responses is warranted.
\end{abstract}

\section{Introduction}

Deep tendon reflex (DTR) tests, such as the patellar reflex test (PTR), are important examinations for identifying the presence and severity of potential neurological disorders [1-4]. For example, detection of depressed and hyperactive responses using DTR tests suggests possible peripheral and central nervous system compromise, respectively [5]. Additionally, these tests are widely used because they are simple and can be performed quickly to assess patients in clinical practice. Despite the common use and simplicity of DTR tests, however, clinical assessments of DTR responses remain subjective and qualitative $[1,6]$. Though some studies have evaluated the subjective assessment and concluded strong correlations with biomechanical and electromyographic measures, other studies have demonstrated low interrater reliability when assessing DTR responses $[7,8]$. Therefore, an opportunity exists to improve the widespread consistency and efficacy of these tests using quantitative methods.
Previous studies have attempted to identify quantitative measures for improving the assessment of PTR tests, which is the focus of this study. The most common variables used to evaluate PTR responses are the first knee extension angle (FKEA) and the tendon reflex amplitude [7,9] which have both demonstrated to correlate well with subjective ratings of the NINDS and Mayo scales [7]. Measurements of angular velocity and acceleration, reflex latency, and movement latency have also been used to quantify PTR responses $[7,10]$. These studies have been useful for evaluating possible quantified variables of PTR responses; however, little research has been performed to correlate experimental data to theoretical models and incorporate the entirety of the PTR response in the assessment.

Therefore, the purpose of this study was to correlate entire experimental PTR responses with a second-order system model and produce novel measurement variables that may be used to improve the efficacy of grading PTR responses. Oscillations of limbs during PTR tests behave similarly to 
oscillations of a spring-mass-damper system because the change in angle from the response dampens over time. It was hypothesized that the characteristic variables of a singledegree-of-freedom, second-order spring-mass-damper system, the exponential decay rate and the damped natural frequency, may describe PTR responses.

\section{Materials and Methods}

The experimental procedure was approved by the Institutional Review Board at Trine University in Angola, Indiana. Written consent was obtained from all subjects before testing. A total of 51 subjects consisting of 36 males and 15 females between 18 and 36 years volunteered to participate in the experiment. The mean age, height, and body mass for male subjects were $21.3 \pm 3.8$ years, $1.81 \pm 0.08 \mathrm{~m}$, and $90.9 \pm 17.7 \mathrm{~kg}$, respectively. Female subjects had a mean age of $20.5 \pm 3.6$ years, mean height of $1.67 \pm 0.09 \mathrm{~m}$, and a mean body mass of $67.8 \pm 11.8 \mathrm{~kg}$. All volunteers who reported any past damage that may affect their nervous system were excluded from the experiment to prevent any abnormal reflex responses. A buck reflex hammer was used to manually elicit responses by tapping the patellar tendon which has been shown to produce similar responses as more complicated instrumental stimulators [11].

Two reflective markers were attached to the sides of two elastic bands each to expedite data collection. Before the experiment, one elastic band was adjusted on the subject's knee so that one reflective marker referenced the lateral tibial condyle and another referenced the medial tibial condyle. The other elastic band was adjusted on the subject's ankle so that one reflective marker referenced the lateral malleolus of the fibula and another referenced the medial malleolus of the tibia. Vicon Nexus software and eight motion capture cameras (Vicon, Oxford, UK) were used to capture the responses at a sampling frequency of $200 \mathrm{~Hz}$.

Each subject sat upright on a high table with both legs hanging off the edge. The tapping location for the patellar tendon was found to elicit the greatest reflex response, as described previously $[3,4]$. The manual tapping angle of approximately $60^{\circ}$ with respect to the patellar tendon was used for all subjects to elicit an adequate reflex response $[3,4]$. All subjects were asked to perform a Jendrassik maneuver by closing their eyes, hooking their fingers together, and forcefully pulling each arm against the other [12]. All subjects performed the maneuver to record the reflex response data consistently. Each tendon was tapped prior to recording reflex responses with approximately the same force. Once recording, the tendon was tapped 5-10 times with each tap applied after the prior response ceased. The reflex found for each subject's knee with the greatest maximum extension angle was used to represent the knee in data analysis [7].

During a typical PTR response, the lower leg oscillates in the sagittal plane and the oscillations dampen until the leg returns to equilibrium. Some motion does occur in the coronal plane; however, the motion is minimal and was neglected to assume a single-degree-of-freedom system. The PTR was therefore modeled as a single-degree-of-freedom, second-order spring-mass-damper system since the extension angle from the response dampens over time. The governing equation of the second-order spring-mass-damper system is given by

$$
\begin{aligned}
\ddot{\theta}(t)+2 \zeta \omega_{n} \dot{\theta}(t)+\omega_{n} \theta(t) & =\frac{f(t)}{m}, \\
\omega_{n} & =\sqrt{\frac{k}{m}}, \\
\zeta & =\frac{b}{2 m \omega_{n}},
\end{aligned}
$$

where $\theta(t)$ is the extension angle as a function of time, $\omega_{n}$ is the undamped natural frequency, $\zeta$ is the damping ratio, $f(t)$ is the input force function, $m$ is the mass, $k$ is the stiffness, $b$ is the damping coefficient. The impulse response of a single-degree-of-freedom, second-order spring-massdamper system is governed by the solution

$$
\theta(t)=\theta_{0} e^{-\lambda t} \sin \left(\omega_{d} t\right)=\theta_{0} e^{-\zeta \omega_{n} t} \sin \left(\omega_{d} t\right),
$$

where $\theta_{0}$ is the angle at initial time and the damped natural frequency is

$$
\omega_{d}=\omega_{n} \sqrt{1-\zeta^{2}}
$$

The solution may be characterized by the exponential decay, $\lambda$, and damped natural frequency, $\omega_{d}$. Since these two characteristics define a single-degree-of-freedom, secondorder spring-mass-damper system, the PTR responses were assumed to be characterized by them as well.

The extension angle was plotted with respect to time for each response and only oscillation peaks greater than $5 \%$ of the maximum extension angle were selected to reduce error caused by the friction of the system independent of displacement and velocity, otherwise known as Coulomb friction. The time period, $T$, between the first and second peaks was used to calculate the damped natural frequency, $\omega_{d}$ :

$$
\omega_{d}=\frac{2 \pi}{T}
$$

The exponential decay equation was also used with the peak angles and times found from the experiment to curve-fit the data:

$$
\theta(t)=\theta_{0} e^{-\lambda t}=\theta_{0} e^{-\zeta \omega_{n} t}
$$

By fitting the exponential decay curve with the experimental oscillation peaks, constants that produced the least error were found for the exponential decay rate, $\lambda$, and the initial extension angle, $\theta_{0}$. The validity of modeling the reflex response as a single-degree-of-freedom, second-order spring-massdamper system was tested with regression analysis between the experimental data and the characteristic variables and presented using the coefficients of determination, $R^{2}$. Ranges of the characteristic measurement variables for the sample population were also presented. 


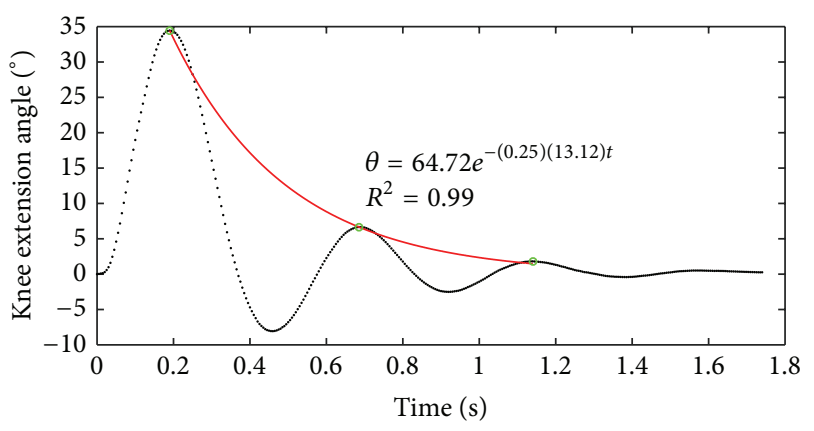

$$
\begin{aligned}
& \text { Experiment } \\
& - \text { Exponential decay }
\end{aligned}
$$

FIGURE 1: Sample patellar reflex response with the oscillation peaks of the extension angle identified and best-fit, exponential decay curve found.

\section{Results}

Six of the subjects were excluded for the analysis because they experienced twitching that caused irregular PTR responses that could not be evaluated, which resulted in analysis of 45 total subjects. Once identified, oscillation peaks were used to calculate the exponential decay rate and damped natural frequency before plotting the best-fit, exponential decay curve (Figure 1). The mean $R^{2}$ value found between the PTR responses and the exponential decay equation was 0.99 with a minimum value of 0.88 .

The 90 total PTR responses were plotted in the complex plane using the exponential decay rate and damped natural frequency found for each. The positive real and imaginary terms of the complex pairs were plotted in the complex plane. The ranges for the exponential decay rate and damped natural frequency found from the evaluated population were -5.61 to -1.42 and $11.73 \mathrm{rad} / \mathrm{s}$ to $14.96 \mathrm{rad} / \mathrm{s}$, respectively. A range of values for the sample population was then calculated using the standard deviations of the exponential decay rate and damped natural frequency (Figure 2).

\section{Discussion}

The most important result of this study was that the experimental PTR responses strongly correlated with the singledegree-of-freedom, second-order spring-mass-damper model. Additionally, the PTR responses were accurately represented by novel measurement variables, the exponential decay rate, and damped natural frequency. Though these response variables are more difficult to conceptually understand than FKEA or angular acceleration and velocity individually, they have been demonstrated to accurately represent PTR responses. The significance of these measurement variables is questionable, as they are not common variables used to describe reflex response; however, the strong correlation of the model using these variables deserves further investigation to determine the usefulness. Use of the measurement variables evaluated in this study may be advantageous for future clinical assessment because they represent all movement of

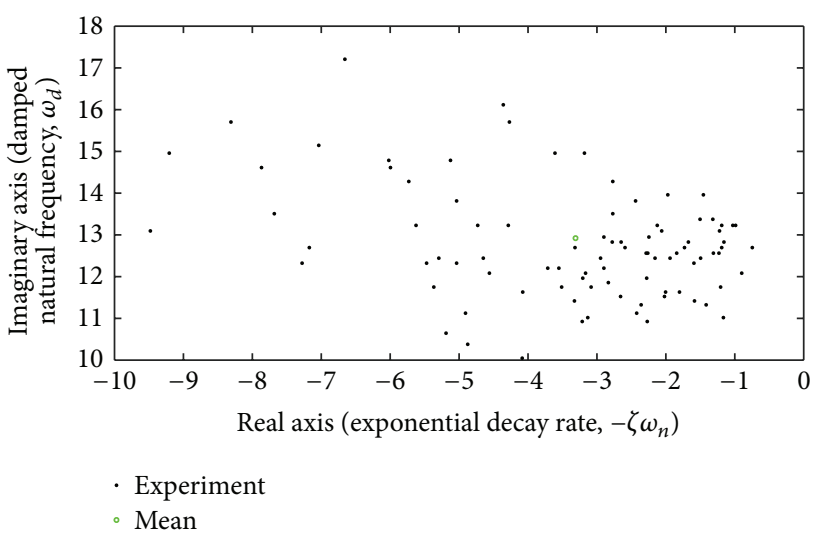

FIGURE 2: Ranges of the exponential decay rate and damped natural frequency determined for the sample population.

the joint throughout the entire response as shown by the solution of the governing equation (2). The exponential decay rate and undamped natural frequency account for the initial PTR response, like previous measurement variables, and for all movement for the remainder of the PTR response. Therefore, the advantage of using the novel measurement variables evaluated in this study is the ability for these variables to represent more information of a single PTR response than previous quantitative methods.

We also recognize that this study has limitations. The range of values for the sample population was presented; however, there was no correlation with clinical assessment so the usefulness of these measurement variables is yet to be determined. Future research should be performed with a wide range of ages and clinical assessments to evaluate the efficacy of the proposed measurement model. In addition, the proposed measurement method is currently unacceptable in clinical settings. For the analysis, motion capture cameras and analysis software were utilized to determine the measurement variables. It is impractical for motion capture cameras and postprocessing software to be available in most clinical setting. Therefore, another future direction of this work will target the development of wearable technology to quickly and easily report the measurement variables. Finally, the assumption to ignore Coulomb friction was a likely source of measurement error. In the theoretical model, excluding oscillation peaks less than $5 \%$ of the maximum extension angle was done to reduce errors attributed to Coulomb friction. The experimental results may be better-fit to theoretical solutions if Coulomb friction is included in the spring-mass-damper model. Future work should also be done using optimization techniques to include Coulomb friction into the analysis of the PTR responses.

\section{Conclusions}

This study confirmed that PTR responses strongly correlate to a single-degree-of-freedom, second-order spring-massdamper model. Additionally, the exponential decay rate and undamped natural frequency were demonstrated to be novel measurement variables to accurately measure PTR responses. 
Therefore, further investigation of these measurement variables and their usefulness in grading PTR responses is warranted.

\section{Conflict of Interests}

The authors declare that there is no conflict of interests regarding the publication of this paper.

\section{Acknowledgments}

The authors would like to thank Mitch Zorger and Neal Lammers from Trine University and Dr. Kyle Steineman from National University of Health Sciences for assisting in the research design. They would also like to thank faculty and students from Trine University who volunteered to participate.

\section{References}

[1] S. Manschot, L. van Passel, E. Buskens, A. Algra, and J. van Gijn, "Mayo and NINDS scales for assessment of tendon reflexes: between observer agreement and implications for communication," Journal of Neurology Neurosurgery and Psychiatry, vol. 64, no. 2, pp. 253-255, 1998.

[2] J. Stam, "Physical diagnostics-tendon reflexes," Nederlands Tijdschrift voor Geneeskunde, vol. 143, no. 16, pp. 848-851, 1999.

[3] L. K. Tham, N. A. Abu Osman, W. A. B. Wan Abas, and K. S. Lim, "The validity and reliability of motion analysis in patellar tendon reflex assessment," PLoS ONE, vol. 8, no. 2, Article ID e55702, 2013.

[4] L. K. Tham, N. A. A. Osman, W. A. B. W. Abas, and K. S. Lim, "Motion analysis of normal patellar tendon reflex," Canadian Journal of Neurological Sciences, vol. 40, no. 6, pp. 836-841, 2013.

[5] G. L. Marshall and J. W. Little, "Deep tendon reflexes: a study of quantitative methods," Journal of Spinal Cord Medicine, vol. 25, no. 2, pp. 94-99, 2002.

[6] N. Mamizuka, M. Sakane, K. Kaneoka, N. Hori, and N. Ochiai, "Kinematic quantitation of the patellar tendon reflex using a triaxial accelerometer," Journal of Biomechanics, vol. 40, no. 9, pp. 2107-2111, 2007.

[7] C. Dafkin, A. Green, S. Kerr, D. Veliotes, and W. McKinon, "The accuracy of subjective clinical assessments of the patellar reflex," Muscle \& Nerve, vol. 47, no. 1, pp. 81-88, 2013.

[8] Y. Kim and J. Weon, "Inter-rater reliability of quantitative knee tendon reflex test for healthy subjects," Physical Therapy Korea, vol. 14, pp. 57-63, 2007.

[9] Y.-W. Kim, "Clinical availability of the deep tendon reflex test using a novel apparatus in healthy subjects," Journal of Physical Therapy Science, vol. 27, no. 2, pp. 317-320, 2015.

[10] R. O’Sullivan, D. Kiernan, M. Walsh, T. O’Brien, and Y. Elhassan, "Characterisation of the patellar tendon reflex in cerebral palsy children using motion analysis," Irish Journal of Medical Science, pp. 1-5, 2015.

[11] J. Stam and K. M. Tan, "Tendon reflex variability and method of stimulation," Electroencephalography and Clinical Neurophysiology, vol. 67, no. 5, pp. 463-467, 1987.

[12] H. Walker, Clinical Methods: The History, Physical, and Laboratory Examinations, Butterworths, Boston, Mass, USA, 3rd edition, 1990. 


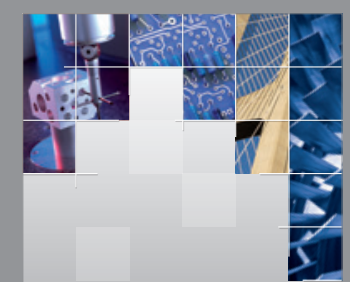

\section{Enfincering}
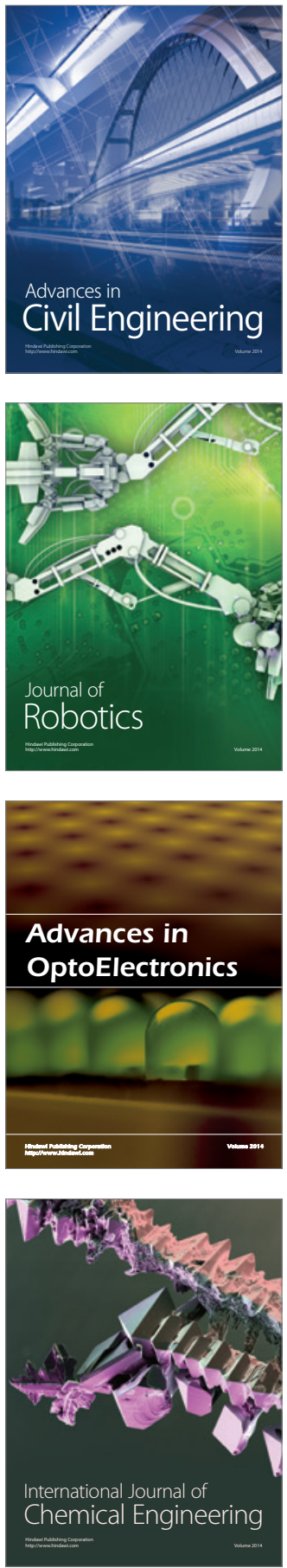

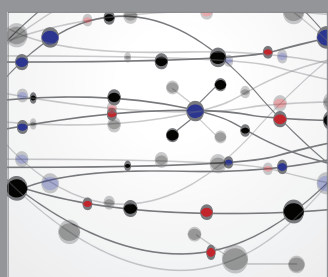

The Scientific World Journal

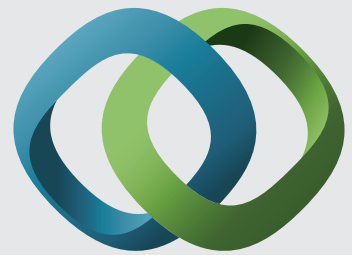

\section{Hindawi}

Submit your manuscripts at

http://www.hindawi.com
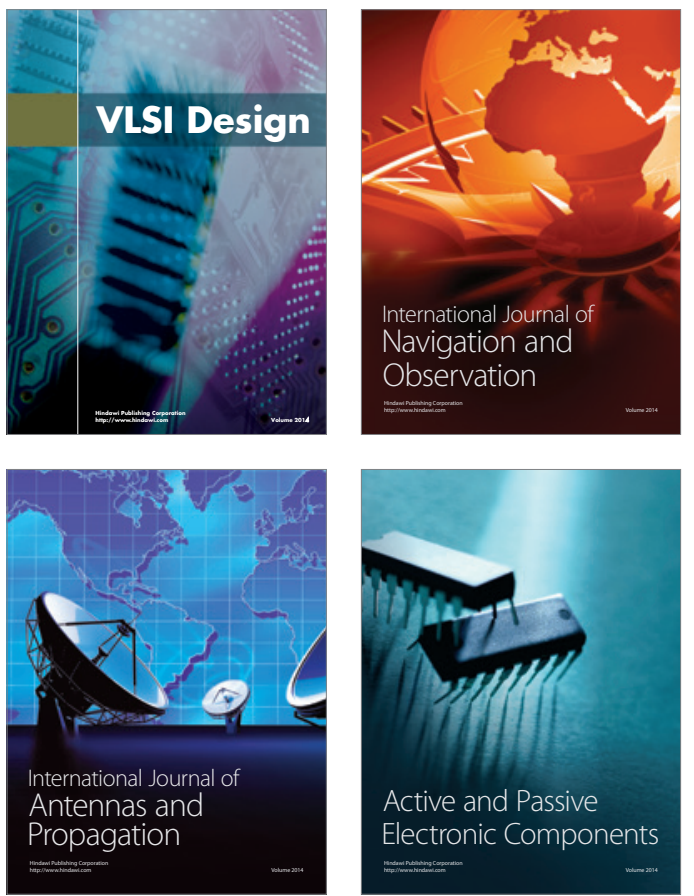
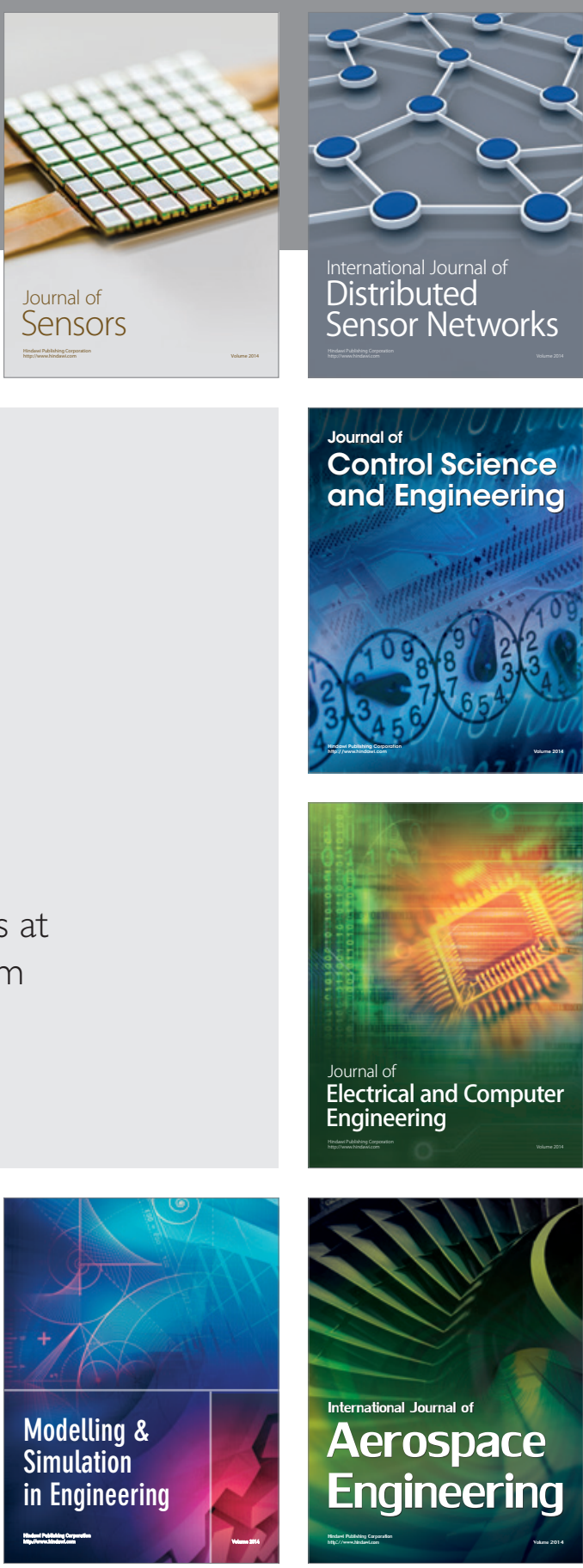

International Journal of

Distributed

Sensor Networks

Journal of

Control Science

and Engineering
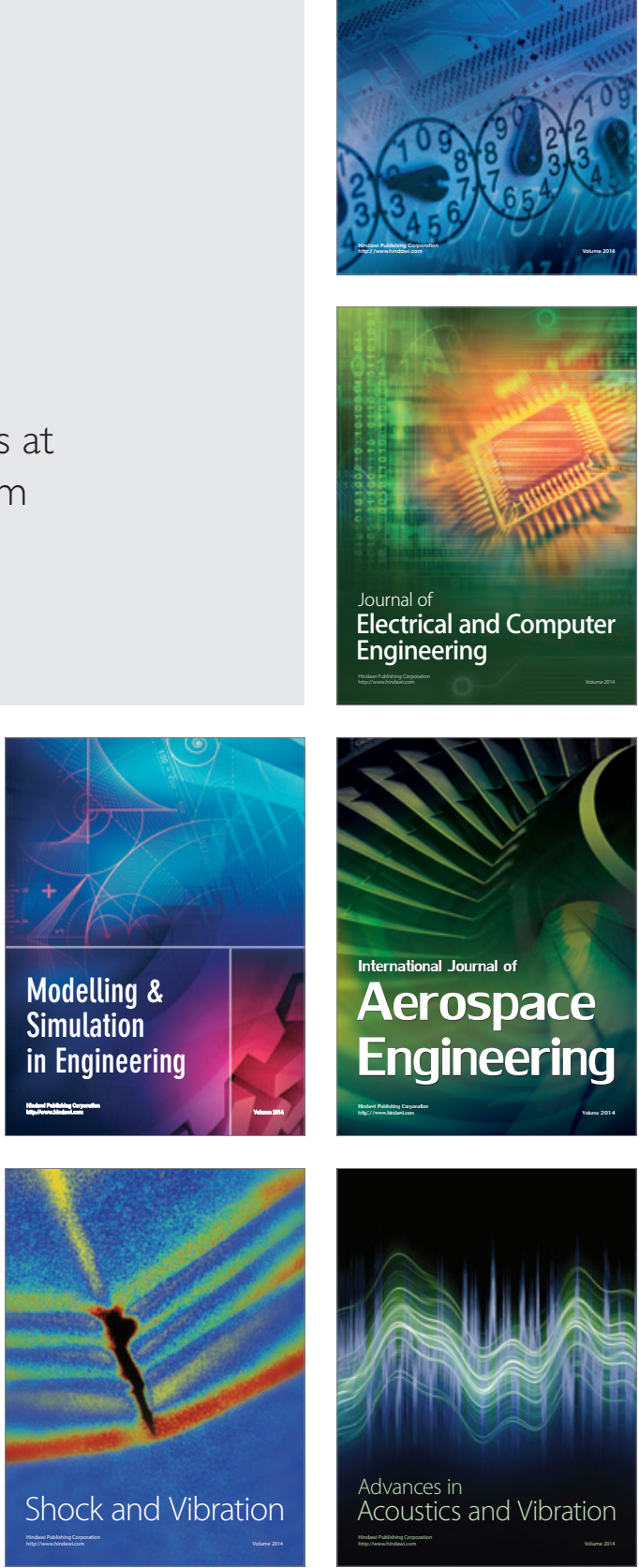